DIGITAL TECHNIQUES 
Macmillan Technician Series

P. Astley, Engineering Drawing and Design II

P. J. Avard and J. Cross, Workshop Processes and Materials I

G. D. Bishop, Electronics II

G. D. Bishop, Electronics III

J. C. Cluley, Electrical Drawing I

J. Elliott, Building Science and Materials

John G. Ellis, and Norman J. Riches, Safety and Laboratory Practice

D. E. Hewitt, Engineering Science II

P. R. Lancaster and D. Mitchell, Mechanical Science III

R. Lewis, Physical Science I

Noel M. Morris, Digital Techniques

Noel M. Morris, Electrical Principles II

Noel M. Morris, Electrical Principles III

Owen Perry and Joyce Perry, Mathematics I 


\title{
DIGITAL TECHNIQUES
}

\author{
Noel M. Morris
}

Principal Lecturer, North Staffordshire Polytechnic 
ISBN 978-1-349-04626-3

ISBN 978-1-349-04624-9 (eBook)

DOI 10.1007/978-1-349-04624-9

(C) Noel M. Morris 1979

Reprint of the original edition 1979

All rights reserved. No part of this publication may be reproduced or transmitted, in any form or by any means, without permission.

First published 1979 by

THE MACMILLAN PRESS LTD

London and Basingstoke

Associated companies in Delhi Dublin

Hong Kong Johannesburg Lagos Melbourne

New York Singapore and Tokyo

Typeset in 10/12 Times

This book is sold subject to the standard conditions of the Net Book Agreement.

The paperback edition of this book is sold subject to the condition that it shall not, by way of trade or otherwise, be lent, resold, hired out, or otherwise circulated without the publisher's prior consent in any form of binding or cover other than that in which it is published and without a similar condition including this condition being imposed on the subsequent purchaser. 


\section{Contents}

Preface

1. The Basis of Logic Systems

1.1 Gates

1.2 Logic Signal Levels

Problems

2. Basic Logic Functions

2.1 The AND Function

2.2 The OR Function

2.3 The NOT Function

2.4 Complete Coin Circuit of the Vending Machine

2.5 Negated Inputs

2.6 The Effect of Positive and Negative Logic Levels

Problems

3. NAND and NOR Networks

3.1 The NAND Function

3.2 The NOR Function

3.3 Why Use NAND and NOR Gates?

3.4 The Effect of Positive and Negative Logic Conventions on NAND and NOR Gates

3.5 NAND Networks

3.6 NOR Networks

Problems

4. Semiconductor Devices

4.1 Semiconductor Materials

4.2 Semiconductor Junction Diodes

4.3 Charge Carrier Storage in Diodes
4.4 The Schottky Barrier Diode

4.5 The Bipolar Junction Transistor (BJT)

4.6 Insulated-gate Field-effect Transistors

Problems

5. Semiconductor Logic Circuits and Integrated Circuits

5.1 Diode-resistor Logic (DRL)

5.2 Limitations of DRL Gates 23

5.3 Current-sourcing and Current-sinking Logic Gates

5.4 Fan-in and Fan-out of Logic Gates 24

5.5 A Resistor-Transistor Logic(RTL) NOT Gate 26

5.6 Thermal Considerations 27

5.7 Noise Immunity 27

5.8 Connections of Unused Input Lines 27

5.9 Time Delays in a Bipolar Transistor Switch 28

5.10 Methods of Improving the Switching Speed 29

5.11 Active Collector Loads 30

5.12 The Wired-OR Function 30

5.13 Diode-transistor Logic (DTL) 32

5.14 Transistor-transistor Logic (TTL) 33

5.15 Emitter-coupled Logic (ECL) 36

5.16 MOS Logic Circuits 37

5.17 CMOS Transmission Gates (Bilateral

Switches) and Three-state Logic 38

5.18 Integrated Injection Logic $\left(\mathrm{I}^{2} \mathrm{~L}\right) \quad 39$

5.19 Monolithic Integrated Circuit Construction 39

5.20 LSI, MSI and SSI 40

5.21 IC Packaging 41

Problems $\quad 42$

6. The Algebra of Logic 43

6.1 The Laws of Logic 43

6.2 Logic Theorems 44

6.3 Applications of the Laws and Theorems of Logic

Problems 


\section{Logic System Design}

7.1 Combinational Logic and Sequential Logic Networks

7.2 The Design of Logic Networks From Truth Tables

7.3 Replacing AND-OR Networks by NAND Networks

7.4 Replacing OR-AND Networks with NOR Networks

7.5 Wired-OR Networks

7.6 The Exclusive-OR Function

7.7 Karnaugh Maps

7.8 Logic Circuit Design Using Data Selector Logic (multiplexer logic) Problems

\section{Flip-Flops}

8.1 Static and Dynamic Memories

8.2 The S-R Flip-flop

8.3 The Gated S-R Flip-flop

8.4 Contact Bounce Eliminators

8.5 Master-Slave Flip-flops

8.6 The Master-Slave J-K Flip-flop

8.7 The Trigger (T) Flip-flop

8.8 The D Master-Slave Flip-flop

8.9 Edge-triggered Flip-flops

8.10 Dynamic Memories

Problems

9. Binary Codes and Arithmetic Processes

9.1 The Binary System

9.2 Fractional Numbers

9.3 Binary Addition

9.4 Addition Networks

9.5 Binary Subtraction

9.6 Negative Numbers

9.7 Multiplication
9.8 Binary Division

9.9 Binary-decimal Codes

9.10 Error Detection Problems

10. Counter and Shift Register Circuits

10.1 A Pure Binary Asynchronous Counter

10.2 A Bidirectional Pure Binary Asynchronous Counter

10.3 An 8421 BCD Asynchronous Counter

10.4 A Reason for Synchronous Counters

10.5 A Synchronous Pure Binary Counter

10.6 A Reversible Synchronous Pure Binary Counter

10.7 A Synchronous 8421 BCD Counter

10.8 Shift Registers and Ring Counters

10.9 A Serial-input, Serial-output Shift Register

10.10 Dynamic Shift Registers

10.11 Ring Counters

10.12 Chain Code Generators

10.13 A Serial Binary Adder

10.14 A Serial Binary Subtractor Problems

\section{Storage Devices and Systems}

11.1 Types of Memory

11.2 Static RAMs

11.3 Dynamic RAMs

11.4 Content Addressable Memories (CAMs)

11.5 Read only Memories (ROMs)

11.6 Programmable Logic Arrays (PLAs)

11.7 Storage of Data on Magnetic Medium

11.8 Ferrite Core Storage

11.9 Reading From and Writing Into a Ferrite Core

11.10 Addressing a Location in a Core Store

11.11 Magnetic Surface Recording

11.12 Methods of Recording Data on a Magnetic Surface 
11.13 Magnetic Bubble Device (MBD) Memories

11.14 Charge-coupled Device (CCD) Memories Problems

Solutions to Selected Problems
102

103

104

105 


\section{Preface}

The explosive growth of digital electronics has affected the life of almost everyone. This book provides coverage of aspects of digital technology ranging from basic gates to the design of digital systems.

Digital technology is wide-ranging and this fact is reflected in TEC Certificate and Diploma courses as well as in TEC Higher Certificate and Diploma courses. The aim of the book is to provide a sound introduction to Digital Techniques associated with these courses. Students attending other courses which include digital technology will also benefit from reading the book.

The eleven chapters provide a solid introduction to digital technology beginning with chapters on basic logic functions and gates, leading to the types of semiconductors used and to integrated circuit technology. A wide range of logic families are described including DTL, TTL, ECL, MOS and $\mathrm{I}^{2} \mathrm{~L}$. There follows two chapters devoted to Boolean algebra and to the use of Karnaugh maps, which form the basis of many design studies. Binary arithmetic methods are introduced in chapter 9, together with binary codes and error detection. The ubiquitous pocket calculator and the microprocessor could not operate without counters, shift registers and storage systems and these are introduced in chapters 10 and 11 . Included in the final chapter are semiconductor storage devices including RAMs and ROMs together with ferrite ring core stores, magnetic bubble devices and charge coupled devices.

I would like to acknowledge the assistance, tolerant understanding and encouragement of my wife during the preparation of this book.

NOEL M. MORRIS 